Canadian Oncology

Nursing Journal

Revue canadienne

de soins infirmiers

en oncologie

Volume 29, Issue 1 - Winter 2019

elSSN: 2368-8076 


\section{The nurse practitioner role is ideally suited for palliative care practice: A qualitative descriptive study}

\author{
by Carmel M. Collins and Sandra P. Small
}

P alliative care (PC) is an approach to caring for individuals with life-threatening health conditions, with the focus being best quality of life (Hawley, 2017). It involves effective symptom management to address the physiological manifestations of diseases; interventions to promote the social, spiritual, and emotional well-being of patients and their families; and end-of-life (EOL) care to support patients' greatest comfort and dignity when death is imminent (World Health Organization, 2011; Worldwide Palliative Care Alliance [WPCA], 2014). Palliative care is recommended not only for cancer, but for any chronic life-threatening condition; for example, heart and kidney failure and various neurological diseases (Hawley, 2017). With the high prevalence and expected growth of chronic diseases not only in Canada, but worldwide, the need for PC is great now and will become even more so in the future (Canadian Hospice Palliative Care Association [CHPCA], 2014; Public Health Agency of Canada, 2016; WPCA, 2014).

Although early integration of PC with disease management is desired for chronic life-threatening conditions, unfortunately, that approach is not often realized (Hawley, 2014). In fact, many individuals who would benefit from PC either do not receive it at all or receive it solely at the end stage of life (Hawley, 2017). In Canada, only up to $30 \%$ of individuals who die receive PC (CHPCA, 2014). Several factors contribute to the accessibility problem: limited availability of PC specialists, that is, health professionals (e.g., physicians, registered nurses [RN]), who have specialty education and clinical experience in the field; inadequate educational preparation of generalist practitioners (e.g., family physicians, community health $\mathrm{RNs}$ ) for palliative practice; insufficient financial resources

\section{ABOUT THE AUTHORS}

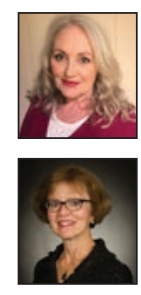

Carmel M. Collins, RN, MN, NP F/AA, CHPCN(C), Nurse Practitioner, Regional Palliative Care Leadership Team, Eastern Health, St John's, NL

Sandra P. Small, RN, PhD, Professor, School of Nursing, Memorial University of Newfoundland, St. John's, NL, A1B 3V6 Tel: 709 777-6973 Fax: 709 777-7037

Email: ssmall@mun.ca

Corresponding Author: Carmel M. Collins, 100 Forest Road, Room 328 Southcott Hall, St. John's, NL, A1A $1 E 5$

Tel: (709) 777-8039

Email: carmelduggancollins@gmail.com

Conflict of Interest

The authors declare that there is no conflict of interest.

Funding

This study was funded through awards from the School of Nursing, Memorial University of Newfoundland and the Association of Registered Nurses of Newfoundland and Labrador.

DOI:10.5737/2368807629149 for palliative services; and rural or remote patient location (CHPCA, 2014; Canadian Society of Palliative Care Physicians, 2016; Hawley, 2017).

It is suggested in the literature that nurse practitioners (NPs) could help fill the gap in PC services (Deitrick et al., 2011). NPs are RNs with additional education and clinical experience (Canadian Nurses Association [CNA], 2016; Canadian Nurse Practitioner Initiative, 2006). As advanced practice nurses (APN), they have the autonomy and competencies to diagnose conditions, order and interpret diagnostic tests, prescribe medications, perform advanced clinical procedures, and manage illnesses, while working within a framework of inter-professional collaboration and patient-centred holistic nursing care. NPs practise in a variety of healthcare settings, including hospitals, community clinics, and LTC facilities, and in urban, rural, and remote locations. Thus, aside from NPs who might be PC specialists or working specifically in PC settings, NPs, more generally, seem well pointed to provide care to individuals with chronic life-threatening conditions, including at advanced stage. Hence, the purpose of this study was to address the question, "What role do NPs who are not PC specialists and not working in palliative specialty settings play in the provision of PC?"

\section{LITERATURE REVIEW}

Little is known about NP practice in PC outside of palliative specialty settings. However, research findings indicate that within palliative settings, NPs contributed to PC through various role functions, including managing medical conditions, consulting with other care providers about patient conditions, providing psychosocial support (e.g., counselling) and education (e.g., about medical condition and treatment) to patients and families, coordinating patient services, providing ongoing care, and advocating for patients (Deitrick et al., 2011; Mitchell et al., 2016; O'Connor, Palfreyman, Le, \& Lau, 2016; Osborn \& Townsend, 1997; Williams \& Sidani, 2001).

Although few studies were found on patient outcomes with NP practice in PC, it seems that NP role functions were conducive to positive results. Healthcare providers in LTC facilities thought that NPs had improved the availability and timeliness of PC interventions and, as a consequence, fewer residents were transferred to hospital for such care (Kaasalainen et al., 2013). Similarly, nurses, physicians, and other stakeholders thought that an NP-led PC service resulted in enhanced care for terminally ill patients, including fewer hospitalizations and more at-home deaths and better support for patients' carers at home (Mitchell et al., 2016). An NP in an oncology PC clinic noted improvement in patient knowledge of and coping with the illness and in patient quality of life, patient adherence to the prescribed plan of care and satisfaction with care, adequate symptom control, and prevention of complications (Williams \& Sidani, 2001). 
The qualitative findings of those studies are consistent with the findings of evaluations of NP palliative practice. A prospective examination of one NP's role in PC within a hospital and a community service indicated that the NP's practice contributed to fewer emergency department (ED) visits and hospital admissions and shorter hospital stays for palliative patients (O'Connor et al., 2016). Feedback from patients and their home carers indicated that the NP's care improved their confidence in managing at home. In another study, based on a review of medical records, a NP's practice in a primary PC clinic was determined to have resulted in fewer patient visits to the ED and improved symptom scores for some patients (Owens et al., 2011).

Although NPs may make a difference for palliative patients, it is important to note that not all NPs may be comfortable (Tyree, Long, \& Greenberg, 2005) or confident (Dahlin, Coyne, \& Cassel, 2016; Letizia \& Jones, 2012) in providing palliative interventions. In a large study of NPs from a variety of practice areas, whereby their involvement in EOL care was examined, the majority thought that having more EOL education and more work experience would enable them to better communicate with patients and families about EOL matters (Tyree et al., 2005). In another study, APNs, including NPs, rated their confidence in $\mathrm{PC}$ provision as low prior to participating in an intensive PC continuing education (CE) program. After the CE, their confidence was significantly improved (Dahlin et al., 2016). Similarly, $61 \%$ of NPs working in LTC homes reported having little to no confidence in evaluating and managing $\mathrm{PC}$ patients prior to participating in a PC CE program. After the CE, $83 \%$ felt confident in those areas (Letizia \& Jones, 2012). Of particular note is that APNs, including NPs, indicated having been exposed to little PC content in their graduate education (Dahlin et al., 2016; Letizia \& Jones, 2012). Likewise, faculty across a large number of graduate programs, while acknowledging the importance of EOL content in graduate education, rated their programs as only moderately adequate and effective, at best, with respect to such content (Paice et al., 2006a, 2006b).

\section{METHODS AND PROCEDURES}

This study was conducted using qualitative description methods, as proposed by Sandelowski (2000). Qualitative description requires the collection of narrative data to fully describe the research topic from the perspective of the study participants. The study was approved by a research ethics board and informed consent was obtained from all participants.

\section{Participants}

The study took place in a province in eastern Canada. Participants of interest were licensed NPs whose main focus was not palliative practice and might or might not have palliative patients in their practices. NPs who were PC specialists or were working in palliative specialty settings were not eligible. The NPs were recruited through information about the study sent by email to all eligible NPs within a defined geographical region, which yielded 11 participants and, through snowball sampling, yielded a further eight participants. Of the 19 NPs, 16 were from an urban area and three were from rural settings.
One NP was a man and 18 were women. The NPs' ages ranged from 32 to 62 years $(x=48.5)$. Seventeen NPs were in family/ all ages practice (i.e., primary care focused on all populations across the lifespan and on prevention and illness) and two were in adult practice (i.e., focused on adults with illness). Five and seven NPs, respectively, had RN diplomas only or nursing baccalaureate degrees prior to their NP preparation and seven NPs had nursing master's degrees specific for NP practice. The NPs had been RNs from 7 to 41 years $(x=26.1)$ and had been NPs from four months to 18 years $(\mathrm{x}=9.4$ years).

The NPs formed two distinct groups with respect to PC: 13 encountered palliative patients in their practices and provided care to them and six did not have contact with palliative patients. The NPs who provided PC practised in community primary health clinics or an ED, providing care to a variety of patients, including patients with chronic diseases and patients with life-threatening conditions, or practised in LTC facilities, providing care to geriatric patients. Those NPs had varied opportunity for PC practice, from minimal to frequent. The NPs who did not have contact with palliative patients practised either in a hospital acute in-patient unit or in specialty clinics where the patient populations did not include palliative patients.

\section{Data Collection and Analysis}

The data were collected by the first author who is a specialist PC NP. Prior to beginning data collection, she reflected upon her knowledge, thoughts, and feelings about the role of the NP in PC and recorded her reflections in a research journal. She periodically reviewed her reflections, as she gathered and analyzed the data in an effort to avoid prejudging or forcing interpretation of the data. One comprehensive semi-structured interview was completed with each NP, using open-ended questions to obtain information about the NPs' experiences, perspectives, and insights in relation to NP practice in PC. Examples of questions are "When I say palliative care what comes to your mind?" "What are your thoughts on the role of the NP in palliative care practice?" Prompting questions and comments were used to elicit detail. The interviews occurred in private, were digitally recorded, and were transcribed verbatim. Seventeen of the interviews were in person and two were by telephone. After each interview, the interviewer noted in her research journal observations she had made about the interview and thoughts she had about what the NP had shared. These reflections informed subsequent interviews and helped the investigators think about what was in the data during the analysis.

The analysis was conducted by applying the procedures of qualitative content analysis (Graneheim \& Lundman, 2003; Sandelowski, 2000), with the two investigators working collaboratively together. The analysis occurred concurrently with data collection. That approach permitted the investigators to see what was in each interview and what needed to be raised in subsequent interviews for the most complete data. The data from each interview were coded and categorized. The categories were synthesized across the interviews to produce a final set of themes, with thematic saturation occurring 
at 19 participants. The analytic process was facilitated by constantly comparing codes and categories within and across interviews, writing memos with illustrative participant voice (using pseudonyms for larger quotations to distinguish participants), and diagramming to explicate the themes, culminating in Figure 1.

\section{RESULTS}

The findings yielded a central theme, namely, the NP role is "ideal[ly]" "suited" for PC practice. As Bertha said, PC practice is "the perfect place for a nurse practitioner." ... "a perfect fit." For some NPs, that view was based on their professional experience of having cared for palliative patients in their NP practice; for the others, who did not have such experience, it was based on their knowledge of "the nature of the nurse practitioner" (Bertha) by virtue of being NPs themselves. The NPs thought that there are factors that facilitate and factors that impede the NP role being ideally suited to PC practice.

The facilitators are attributes of the NP role and are represented by three themes:

1. Broad Scope and Autonomous Practice - The NPs knew that palliative patients have complex medical care needs and they were especially attuned to the importance of "comfort measures ... and pain control" (Emma). They highlighted several specific features of NPs' broad "scope of practice" and "autonomy" that make the role ideally suited to meeting such needs for palliative patients' "best quality of life" (Kate). The features go well beyond what staff RNs are able to do and include having advanced skills and knowledge to assess, diagnose, and treat medical conditions and being able to intervene for symptom management, prescribe medications (especially controlled drugs such as opioids), manage all aspects of care, and provide continuing care. That NPs are suited to meeting the comprehensive needs of palliative patients was intimated by Rita: "It would be so good to have a nurse practitioner who could go and visit [the palliative] person and look after all the needs whatever they may be, it would be ideal really."

2. Situated Practice - The NPs reasoned that NP practice is

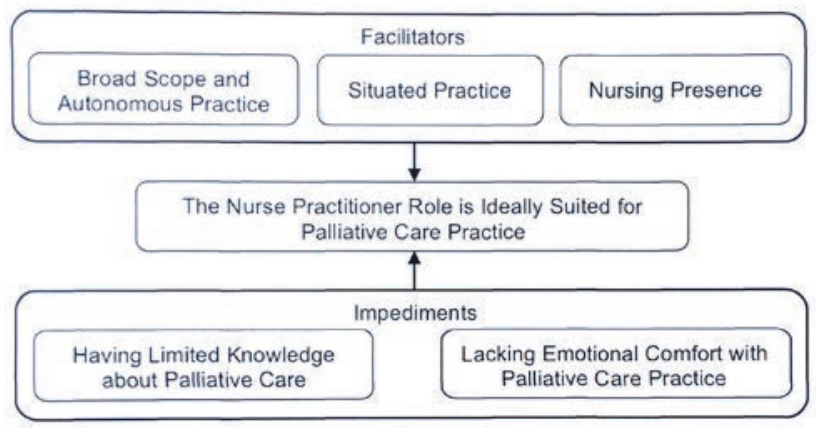

Figure 1: Thematic representation of facilitators of and impediments to the NP role being ideally suited for PC practice. Facilitators are the NP role attributes of broad scope and autonomous practice, situated practice, and nursing presence. Impediments are the NP personal attributes of having limited knowledge about PC and lacking emotional comfort with PC practice. situated for PC in that patients who need such care are found in many inpatient and outpatient settings in which NPs work, aside from specialty palliative and oncology settings. They drew attention to geriatric LTC and rural primary healthcare as practice areas where NPs are especially likely to encounter patients requiring PC. Geriatric LTC facilities routinely have palliative patients because of the prevalence of chronic life-threatening diseases in the elderly residents. In rural locations, specialty PC services are generally not available, leaving patients to rely on their primary healthcare practitioner for their PC needs. In geriatric LTC facilities and rural settings, an NP may be the main or sole healthcare provider. Such opportune work settings situate NPs for PC practice. Sarah described the unique situation of NPs in rural primary healthcare:

Especially in the rural areas where there is no team. ... You are the team. ... the care provider. ... put the plans in place. ... follow them [palliative patients] through all the stages ... titrat[e] medications. ... provid[e] the education and the psychosocial support.

3. Nursing Presence - The NPs believed that nursing "presence" with patients and their families is essential in PC. They further believed that NPs have the opportunity and competencies for presence and their holistic approach embodies it, making them ideally suited for PC practice. Being present is about being available, in attendance, and attentive. It is about "being there, just absolutely being there." (Lynn) NPs are available and in attendance by virtue of their direct role in providing ongoing care. They are attentive through meeting physical and psychosocial needs to optimize patient well-being. Presence is enabled by NPs' "advanced communication" and "active listening" skills and capacity for "empathy." The NPs emphasized the importance of presence to the development of a "trust" relationship between the healthcare provider and palliative patient and family, which is particularly important to having open and honest conversations about the illness, prognosis, and EOL care and, ultimately, to helping patients and families find a psychological "place of comfort" (Mary). As Sally conveyed, presence makes it possible to talk with patients about their "spirituality, their faith, their family support. ... Have they accomplished what they needed to accomplish in this life? Do they feel that they're missing something ... or are they just sick of being sick and they want it over?"

The impediments that detract from the ideal suitability of the NP role for PC practice are personal attributes of NPs, as represented by two themes:

1. Having Limited Knowledge about Palliative Care - The NPs thought that "specialty" palliative knowledge is required for PC practice. They further thought that without specialty knowledge NPs' knowledge for PC is limited and having limited knowledge interferes with the suitability of the NP role for such practice. The NPs considered that specialty PC knowledge is acquired from formal academic education and $\mathrm{CE}$ in the subject matter and from working in the field. Interestingly, they indicated that their formal NP academic 
education either lacked PC content or did not have sufficient emphasis on NP "management ... of a palliative care patient" (Emma). They thought that "what you've learned in your [NP] program is not enough" (Kate) to feel knowledgeable about providing PC. "As a new NP you really have no concept of ... how to truly care for someone in palliative care and you're nervous" (Kate) about providing such care.

Therefore, it is not surprising that the NPs in this study whose practice did not involve PC patients indicated having little knowledge about PC. Of the NPs who had palliative patients in their practice, some revealed a good knowledge base with respect to PC despite thinking that their formal NP academic education "certainly didn't prepare you for caring for palliative care patients." (Tina) Those NPs attributed their knowledge of $\mathrm{PC}$ to having experience caring for palliative patients over time and working with or consulting with other professionals in their collaborative practice or with specialists in the field. Some of those NPs had participated in PC CE opportunities, such as PC conferences, which they also thought enhanced their knowledge. The NPs emphasized the value of and confidence gained by being able to consult with knowledgeable others for support in their decision-making for the best management of palliative patients. "I could always call up and discuss pain management with the $[\mathrm{PC}]$ team ... I always felt there was someone to call if we were perplexed with some symptom management. ... So that was always a good thing." (Mary) Some NPs suggested that having access specifically to a specialty PC NP would be particularly helpful for mentoring and guidance in PC practice.

The remaining NPs had limited experience as NPs providing care to palliative patients, having encountered only a few in their practices, and indicated having limited knowledge about PC. They recognized that they needed "more information and knowledge" (Marie) about this area of practice, especially with respect to "protocols and clinical guidelines and evidence-based practice" (Sally). For instance, they highlighted prescribing opioids, providing EOL care, and discussing EOL decision-making with patients and families as particular aspects of PC for which they could benefit from further knowledge. Although those NPs valued CE for enhanced practice, they had not participated in PC CE yet. As well, although they viewed consulting with palliative experts as important to feeling confident and certain in palliative practice, some indicated that they did not have access to or were unaware of the availability of any such support.

Whether or not the NPs who provided PC had participated in PC CE, most thought that there is little support in the healthcare system for $\mathrm{CE}$ in general, in terms of covered expenses and leave from work to attend educational functions. For instance, they noted that NPs who are interested in attending CE events might have to use their vacation leave. Because of the limited support in the system, some NPs thought their only means of PC education was independent learning through such sources as journal articles and the internet.

2. Lacking Emotional Comfort with Palliative Care Practice The NPs thought that PC requires a particular kind of person, that is, one who has the emotional comfort needed to work with patients and families who are facing life-threatening conditions that lead to death. Hence, the suitability of the NP role for PC practice is affected by whether or not NPs are at ease with providing PC. The NPs believed that PC practice is "not for everybody" and NPs who are "not comfortable" with PC prefer to work with populations that do not need such care. Indeed, some of the NPs who were not involved in PC practice revealed that they would be uncomfortable with PC practice because of the emotional challenge of working in such sad patient situations.

Conversely, NPs who were involved in PC practice had at least some level of emotional comfort with providing PC. Those NPs had a personal philosophy of dying and death that contributed to their emotional comfort, such as death is a "normal" part of life and "dying is a part of living" (Joan). However, they indicated that their level of comfort was also affected by the extent of their clinical experience in the field. Indeed, NPs with greater experience expressed being fully emotionally comfortable with PC. They thought it a privilege to work with palliative patients and their families, and they derived great satisfaction from it, as Rita revealed:

When you know that you can help somebody have a better experience, it makes a difference. ... It makes you feel good about yourself if you can ... help somebody and make life easier for somebody else; then you know you've done your job.

\section{DISCUSSION}

The NPs in this study were not PC specialists and did not work in palliative specialty settings, and regardless of whether or not they had provided care to palliative patients, all thought the NP role is ideally suited for PC practice. That perception is consistent with findings of other studies whereby NPs were noted as making important contributions to PC (Kaasalainen et al., 2013; Mitchell et al., 2016; O’Connor et al., 2016; Owens et al., 2011). Indeed, authors have concluded that because of NPs' experience and education, they are ideally suited to meet the primary and PC needs of patients at the EOL (Owens et al., 2011) and that NPs may be the best hospital staff to intervene with patients at EOL (Tyree et al., 2005).

The NPs in this study attributed the suitability of the NP role for palliative practice to three features of the role: (a) broad scope and autonomous practice, which permit NPs to treat and manage palliative patients' medical conditions for the patients' best quality of life; (b) situated practice, which provides NPs with opportunity for PC; and (c) nursing presence, which enables NPs' attentiveness to all patient needs, including psychosocial needs, for optimal well-being. Medical management, as a role function of NPs in PC practice, also has been identified in other studies (Deitrick et al., 2011; Kaasalainen et al., 2013; Mitchell et al., 2016; O’Connor et al., 2016; Osborn \& Townsend, 1997). Although little is known about NP practice with palliative patients in settings other than within specialty palliative services, in a study about palliative patients in LTC facilities, findings indicated that NPs were well positioned to offer and promote optimal PC for residents and their families (Kaasalainen et al., 2013). Consistent with the views of the NPs 
in this study that nursing presence is crucial to PC and nursing presence is inherent in NP practice by virtue of NP holistic approach, NPs in another study revealed that through being present with their patients, they were able to provide the very important psychosocial support needed in PC. The NPs spent extended time with their patients, offered counselling, and created an environment for a trusting relationship (Deitrick et al., 2011). That NPs exhibit presence in their practice is also supported by reports of staff and family members in LTC facilities that NPs spent time with palliative families listening to and supporting them (Kaasalainen et al., 2013). Nursing presence has been examined extensively in the literature, as a phenomenon that is foundational to nursing practice (Easter 2000; Finfgeld-Connett, 2006; McMahon \& Christopher, 2011). It is identified in nursing theories as a humanistic quality and way of being in the nurse-patient relationship that enables the nurse to understand patients' unique experiences for individualized patient care (Parse, 1992; Paterson \& Zderad, 1978; Sitzman \& Watson, 2014). Because of its essentiality to nursing, it is perhaps not surprising that NPs acknowledge nursing presence as core to NP practice and demonstrate it in their interactions with patients and families.

Although the NPs in this study thought the NP role is ideally suited for PC practice, they also thought that the suitability of the role for palliative practice is impeded if NPs lack specialty palliative knowledge or emotional comfort with PC. However, regardless of their academic designation $(\mathrm{RN}, \mathrm{BN}$, or $\mathrm{MN})$, the NPs reported having had little, if any, PC content in their formal academic NP education. That NPs may be lacking PC content in their undergraduate or graduate level education has also been reported in other studies (Dahlin et al., 2016; Letizia \& Jones, 2012; Paice et al., 2006a, 2006b). NPs in this study who had good knowledge of PC indicated it was because of their experience in the field, collaborative and consultative practice with knowledgeable professionals, and for some, participation in CE activities. It seems only reasonable then that the NPs who had little experience in PC and had not participated in PC CE or had not had palliative consultative support would have limited palliative knowledge. That those NPs realized needing more PC knowledge is consistent with the findings of other studies. NPs acknowledged needing to know more about PC, having little or no confidence in providing PC (Dahlin et al., 2016; Letizia \& Jones, 2012), and needing to have more EOL education and experience for enhanced communication with patients and families about such care (Tyree et al., 2005). No studies were found about the contribution of consultative support for NPs in PC. However, consistent with the views of NPs in this study that CE is valuable for enhanced PC practice, NPs in other studies who had participated in PC CE had improved confidence in providing PC (Dahlin et al., 2016), comfort in initiating EOL discussions (Tyree et al., 2005), and knowledge about PC and had changed their practice, as a result (Letizia \& Jones, 2012).

The NPs in this study thought that clinical experience is also important for emotional comfort in working with patients and families who are facing death. No studies were found in which NP emotional comfort with PC practice had been examined. However, similar to the finding in this study that some NPs would be uncomfortable with palliative practice because of the emotional challenges in such practice, generalist nurses working in various hospital and community settings, outside of specialty palliative settings, experienced emotional difficulty in providing care to terminally ill patients (Bloomer, Endacott, O'Connor, \& Cross, 2013; Wallerstedt \& Andershed, 2007; Weigel, Parker, Fanning, Reyna, \& Gasbarra, 2007). On the other hand, similar to the NPs in this study who had a philosophy of dying and death that was conducive to emotional comfort with PC and who derived satisfaction from caring for palliative patients, nurses who had chosen to work in PC settings had high commitment to, found purpose in, and demonstrated resilience to the work, which suggest that a particular type of personality may be required for PC (Ablett \& Jones, 2007). Hence, as conveyed by the NPs in this study and supported by the findings of previous studies, PC practice may not be suitable for all nurses.

\section{Study Limitation and Implications for Education, Policy, and Research}

The convenience sample of 19 NPs from a single region within one Canadian province is a limitation of this study. However, the diversity of NPs (varied practice settings, including urban and rural locations; educational backgrounds; and PC experience), along with adherence to procedures for rigorous qualitative descriptive research, strengthen the findings; thus, adding to and supporting the small body of existing knowledge about NPs and PC.

Because of the large and diverse volume of subject matter that needs to be addressed in NP educational programs, it may be unrealistic to expect extensive in-depth specialty PC coverage, as well. However, content about fundamental best practices in PC seems appropriate with, perhaps, a more in-depth focus on PC for NP students who plan to work in areas that have palliative populations, especially LTC, primary care, and specialty PC settings. In addition to the current NP practice streams in educational programs (i.e., family/all ages, adult, pediatric,), educational institutions could consider introducing a PC specialist stream. Furthermore, it is suggested that NPs pursue professional development in an ongoing manner to support palliative practice, such as by participating in formal initiatives (e.g., PC conferences) and informal learning (e.g., accessing academic literature). Those who are engaged in regular palliative practice could consider obtaining specialty certification such as the CNA Hospice Palliative Care Nursing designation. Healthcare institutions should ensure that financial resources are available to facilitate NPs' continuing education endeavours and should ensure that NPs have access to palliative experts for consultation about palliative management of patients under their care.

With adequate educational preparation in PC and emotional comfort in working with palliative patients and families, it seems that NPs would be ideally suited to PC practice and to helping meet the growing need for PC services. However, little research has been conducted on the effectiveness of NPs in PC, both in terms of PC specialists and non-specialists. Hence, more research is required to determine patient and healthcare system outcomes when NPs provide PC. 


\section{REFERENCES}

Ablett, J.R., \& Jones, R.S.P. (2007). Resilience and well-being in palliative care staff: A qualitative study of hospice nurses' experience of work. Psycho-Oncology, 16, 733-740. doi:10.1002/pon.1130

Bloomer, M.J., Endacott, R., O'Connor, M., \& Cross, W. (2013). The 'disease' of dying: Challenges in nursing care of the dying in the acute hospital setting. A qualitative observational study. Palliative Medicine, 27(8), 757-764. doi:10.1177/0269216313477176pmj.sagepub.com

Canadian Hospice Palliative Care Association. (2014). Fact sheet: Hospice palliative care in Canada. Retrieved from http://www.chpca. net/media/330558/Fact_Sheet_HPC_in_Canada\%20Spring\%20 2014\%20Final.pdf

Canadian Nurse Practitioner Initiative. (2006). Nurse practitioners: The time is now-a solution to improving access and reducing wait times in Canada. Retrieved from http://docplayer.net/5707777-A-solution-toimproving-access-and-reducing-wait-times-in-canada.html

Canadian Nurses Association. (2016). The nurse practitioner: CNA position. Retrieved from https:// www.cna-aiic.ca/ / media/cna/page-content/pdf-en/ the-nurse-practitioner-position-statement_2016

Canadian Society of Palliative Care Physicians. (2016). How to improve palliative care in Canada: A call to federal, provincial, territorial, regional and local decision-makers. Retrieved from http://www. cspcp.ca/wp-content/uploads/2016/11/Full-Report-How-toImprove-Palliative-Care-in-Canada-FINAL-Nov-2016.pdf

Dahlin, C., Coyne, P.J., \& Cassel, J.B. (2016). The advanced practice registered nurses palliative care externship: A model for primary health care education. Journal of Palliative Medicine, 19(7), 753-759. doi:10.1089/jpm.2015.0491

Deitrick, L.M., Rockwell, E.H., Gratz, N., Davidson, C., Lukas, L., Stevens, D., ... Sikora, B. (2011). Delivering specialized palliative care in the community: A new role for nurse practitioners. Advances in Nursing Science, 34(4), E23-E36. doi:10.1097/ ANS.0b013e318235834f

Easter, A. (2000). Construct analysis of four modes of being present. Journal of Holistic Nursing, 18, 362-377. doi:10.1177/089801010001800407

Finfgeld-Connett, D. (2006). Meta-synthesis of presence in nursing. Journal of Advanced Nursing, 55(6), 708-714. doi:10.1111/ j1365-2648.2006.03961.x

Graneheim, U.H., \& Lundman, B. (2003). Qualitative content analysis in nursing research: Concepts, procedures and measures to achieve trustworthiness. Nursing Education Today, 24, 105-112. doi:10.1016/j.nedt.2003.10.001

Hawley, P.H. (2014). The bow tie model of 21st century palliative care. Journal of Pain and Symptom Management, 47(1), e2-25. doi:10.1016/j.jpainsymman.2013.10.009

Hawley, P. (2017). Barriers to access to palliative care. Palliative Care: Research and Treatment, 10, 1-6. doi:10.1177/1178224216688887

Kaasalainen, S., Ploeg, J., McAiney, C., Martin, L. S., Donald, F., Martin-Misener, R., ... Sangster-Gormley, E. (2013). Role of the nurse practitioner in providing palliative care in long-term care homes. International Journal of Palliative Nursing, 19, 477-485. doi: 10.12968/ijpn.2013.19.10.477

Letizia, M., \& Jones, T. (2012). An educational intervention for nurse practitioners providing palliative care in nursing homes. Journal of Hospice and Palliative Nursing, 14, 351-357. Retrieved from http:// www.nursingcenter.com/journalarticle?Article_ID=1359588

McMahon, M.A., \& Christopher, K.A. (2011). Toward a midrange theory of nursing presence. Nursing Forum, 46(2), 71-82. doi:10.1111/j.1744-6198.2011.00215.x

Mitchell, G.K., Senior, H.E., Bibo, M.P., Makoni, B., Young, S.N., Rosenberg, J.P., \& Yates, P. (2016). Evaluation of a pilot of nurse practitioner led, GP supported rural palliative care provision. BMC Palliative Care, 15(93). doi:10.1186/s12904-016-0163-y

O'Connor, M., Palfreyman, S., Le, B., \& Lau, R. (2016). Establishing a nurse practitioner model to enhance continuity between palliative care settings. International Journal of Palliative Nursing, 22(12), 581585. Retrieved from http://www.magonlinelibrary.com/toc/ijpn/22/12

Osborn, C.L., \& Townsend, C.H. (1997). Analysis of telephone communication between hospice nurses and a nurse practitioner group. Home Health Care Management a Practice, 9, 52-58. doi:10.1177/108482239700900513

Owens, D., Eby, K., Burson, S., Green, M., McGoodwin, W., \& Isaac, M. (2011). Primary palliative care clinic pilot project demonstrates benefits of a nurse practitioner-directed clinic providing primary and palliative care. Journal of the American Academy of Nurse Practitioners, 24, 52-58. doi:10.1111/j.1745-7599.2011.00664.x

Paice, J.A., Ferrell, B.R., Virani, R., Grant, M., Molloy, P., \& Rhome, A. (2006a). Graduate nursing education regarding end-of-life care. Nursing Outlook, 54, 46-52. doi:10.1016/j.outlook.2005.04.003

Paice, J.A., Ferrell, B.R., Virani, R., Grant, M., Molloy, P., \& Rhome, A. (2006b). Appraisal of the graduate end-of-life nursing education consortium training program. Journal of Palliative Medicine, 9, 353-360. doi:10.1089/jpm.2006.9.353

Parse, R.R. (1992). Human Becoming: Parse's theory of nursing. Nursing Science Quarterly, 5, 35-42. doi:10.1177/089431849200500109

Paterson, J.G., \& Zderad, L.T. (1978). Humanistic Nursing. New York: National League for Nursing.

Public Health Agency of Canada. (2016). How healthy are Canadians? A trend analysis of the health of Canadians from a healthy living and chronic disease perspective. Ottawa, ON: Minister of Health. Retrieved from https://www.canada.ca/content/dam/phac-aspc/ documents/services/publications/healthy-living/how-healthycanadians/pub1-eng.pdf

Sandelowski, M. (2000). Whatever happened to qualitative description? Research in Nursing and Health, 23, 334-340. doi:10.1002/1098-240X(200008)

Sitzman, K., \& Watson, J. (2014). Caring science, mindful practice: Implementing Watson's human caring theory. New York: Springer.

Tyree, T.L., Long, C.O., \& Greenberg, E.A. (2005). Nurse practitioners and end-of-life care: Beliefs, practices, and perceptions. Journal of Hospice and Palliative Nursing, 7, 45-51. Retrieved from http:// journals.lww.com/jhpn

Wallerstedt. B., \& Andershed, B. (2007). Caring for dying patients outside special palliative care settings: Experiences from a nursing perspective. Scandinavian Journal of Caring Sciences, 21, 32-40. doi: 10.1111/j.1471-6712.2007.00430.x/full

Weigel, C., Parker, G., Fanning, L., Reyna, K., \& Gasbarra, D.B. (2007). Apprehension among hospital nurses providing end-of-life care. Journal of Hospice and Palliative Nursing, 9(2), 86-91. doi:10.1097/01. NJH.0000263530.37671.b8

Williams, D., \& Sidani, S. (2001). An analysis of the nurse practitioner role in palliative care. Canadian Journal of Nursing Leadership, 14(4), 13-19. doi:10.12927/cjnl.2001.19131

Wong, S.L., Gilmour, H., \& Ramage-Morin, P.L. (2016). Alzheimer's disease and other dementias in Canada. Health Reports, 27(5), 11-16. Retrieved from http://www.statcan.gc.ca/pub/82003-x/2016005/article/14613-eng.pdf

World Health Organization. (2011). Palliative care for older people: Better practices. Retrieved from http://apps.who.int/iris/ bitstream/10665/107290/1/e95052.pdf

Worldwide Palliative Care Alliance. (2014). Global atlas on palliative care at the end of life. Retrieved from http://www.thewhpca.org/ resources/global-atlas-on-end-of-life-care 